\title{
Prise en compte du risque du sol par l'entreprise
}

\section{J.-C. RAFFARD}

SOGEPROM

Les Miroirs - La Défense 3 92978 Paris La Défense

Cedex
Quand l'entreprise de BTP réalise un ouvrage, elle assure la responsabilité de cet ouvrage, et aussi celle du sol qui le supporte. Cette situation très contraignante et, parfois, hasardeuse est issue de la législation française liée au code civil. Pour y répondre, lentreprise qui en a pris conscience assure un rôle déterminant dans la définition et la réalisation de ses constructions. Elle a orienté ses procédures de fonctionnement afin d'être plus efficace et plus performante.

Mots-clés : entreprise, fondation, étude, constrution.

\section{Soil condition hazards in Building Contractor action}

NDLE: Les discussions suT cet article sont acceptées jusqu'au $1^{\text {er }}$ octobre 2001.
When Building Contractor is constructing a building, he has to be responsible of this building and of its bearing soli. This situation very compellable and sometimes venturous comes from French legal rules as u Code Civil x. In response of this meaning, skill Building Contractors have been taken a determinating place for defining schemes of their projects before execution. By this way Bullding Contractor has orientated his working procedures for efficiency and better results.

Key words : contractor, foundation, project study, building, 


\section{Introduction}

Avant d'entrer dans la logique qu'ont les entreprises de traiter des problèmes de sols, nous devons au préalable camper le décor.

\section{1}

\section{L'entreprise}

Le champ sera ici limité à l'entreprise française de bâtiment, qui a signé un marché de travaux avec un maître d'ouvrage, aux termes duquel elle doit réaliser en production propre ou en les sous-traitant, des travaux de fondations et/ou de dallages.

En revanche, nous n'aborderons pas le cas de l'entreprise de fondations (profondes) ni celui du « dallagiste $x$.

A partir de là, il faut bien admettre que chaque entreprise a sa façon d'aborder ce type de problème. En effet, un certain nombre de facteurs interviennent en fonction de critères de base, dont les suivants :

- dimension de l'entreprise (grande entreprise ou PME);

- culture de l'entreprise:

- sensibilité de ses dirigeants envers la technique : - connaissances et capacités techniques des exécutants ; - conseil du bureau d'études ;

- etc.

L'entrepreneur ne doit jamais oublier que considéré comme "sachant ", il doit user de son " devoir de conseil $x$ auprès de son maitre d'ouvrage.

Et ceci, s'il ne le sait pas, il l'apprend très vite à ses dépens lorsque réalisant ses travaux en respectant le projet imparfaitement conçu par le maitre d'ceuvre, il se voit reconnu (et c'est facile) * responsable w dans un sinistre, et condamné pour une part importante pour sa réparation...

Hé ! oui, l'entrepreneur est le plus solvable des intervenants de la construction!

\section{8}

\section{Le contrat de travaux}

Marché public, marché privé, le comportement de l'entrepreneur n'est ici pas très différent, encore que le maitre d'ouvrage est souvent représenté par son maitre d'cuvre qui devient dans ce cas maître d'ouvrage délégué. Les rapports peuvent dans ce cas revêtir un aspect plus spécifique, dans lesquels le maître d'ouvrage délégué peut peser dans les options constructives.

L'entreprise réalise les travaux de gros ceuvre dans le cadre d'un marché d'entreprise générale ou un marché de gros ceuvre seul. Dans ce dernier cas, elle peut être co-traitante ou co-traitante mandataire.

On distingue également un marché au forfait d'un marché au bordereau.

Suivant le type de contrat, l'entreprise pourra prendre une disposition particulière pour réaliser ses travaux.
Dans certains cas, elle sera amenée à sous-traiter tout ou partie de ses travaux de fondations et/ou de dallages.

\section{2}

\section{Vie d'une affaire}

Une affaire, est la raison de vivre de l'entreprise.

Elle doit être bonne sur les plans de l'économie et de l'image.

Pour réussir un chantier, l'entrepreneur doit le préparer, le réaliser conformément au projet, au planning et pour le prix convenu.

Quand le client réceptionne son ouvrage, il doit être satisfait, et l'entreprise doit s'en assurer,

\section{3}

\section{Étude d'une affaire}

Dès la phase commerciale, l'entreprise doit être vigilante eu égard aux conditions de sol.

En effet, à quoi bon répondre à un dossier en chiffrant la construction d'un ouvrage si on ne s'est pas préoccupé sérieusement de son support... et pourtant !

Une entreprise, digne de ce nom, donnera des consignes à ses ingénieurs d'études de prix pour qu'ils analysent les pièces d'appel d'offres avant d'établir le coût d'une affaire.

Ainsi, elle s'assure de la faisabilité de l'ouvrage ainsi que de la suffisance et de la cohérence, des cahiers des charges techniques généraux et particuliers, rapport géotechnique, plans d'architecte, plans techniques éventuels d'infrastructures et de fondations, des mitoyens, des héberges, du rapport de sécurité, du plan de sécurité, des conditions administratives, etc.

Elle doit prendre conseil dans le cas où elle ne maitrise pas suffisamment la mécanique des sols, pour étudier si une solution plus économique est envisageable.

C'est tout de même là qu'il faut se rappeler un des objectifs de l'entreprise : elle se doit de construire bien et économiquement pour rester compétitive, c'est-à-cire préparer aussi son devenir.

\section{1}

\section{Suffisance et pertinence d'éléments}

L'entreprise doit détecter si, compte tenu des pièces écrites qui lui sont soumises, elle a suffisamment de renseignements pour « ne pas se faire piéger $»$.

En effet, dans des marchés au forfait, il est toujours écrit dans une des pièces, que l'entrepreneur reconnait avoir pris connaissance du terrain, l'avoir visité, et en faire son affaire pour réaliser ses ouvrages sans exiger de supplément de prix au cas où les conditions deviendraient différentes à l'ouverture des fouilles !

La vigilance doit donc être importante pour vérifier que la mission confiée au bureau d'études de sols par le maître d'ouvrage est bien adaptée :

- au projet ;

- à la destination immédiate ou future et à l'usage du projet dans sa durée de vie ;

- à l'évolution des sols... 
Combien de fois ai-je pu avoir entre les mains un rapport de sols avec les résultats de 3 pénétromètres dynamiques et 2 pressiomètres, pour déterminer, des efforts à reprendre dans une paroi moulée.

Malheureusement, cela ne choque que peu de monde!

Un entrepreneur avisé est vigilant. Souvent, il voudra rencontrer l'auteur du rapport de sol, pour prendre conseil, voire commanditer une campagne de sols complémentaire.

Parfois, avec une connaissance plus pertinente des conditions géotechniques, l'entrepreneur se voit amené à prévoir des solutions qui vont peser sur son prix. Si le client est plus attaché à la « moins-disance » qu'à la "mieux-disance $»$, cette affaire sera attribuée à une concurrence souvent innocente et incompétente. Mais que de larmes versera-t-elle par la suite !

Combien de maitres d'ouvraçe désirant réaliser des bâtiments de logistiques, mal conseillés par leurs maitres d'cuvre commandent une étude géotechnique trop sommaire pour définir les paramètres nécessaires à une bonne définition du dallage. De plus, des confusions ou des omissions dans les documents techniques négligent notamment, sa surface, sa charge d'exploitation, sa déformation admissible, ses liaisons avec les structures et les réseaux. Enfin, la prise en compte de l'effet du chargement induit dans les couches profondes est escamotée... Monsieur Boussinesq est complètement oublié !

\section{... L'entrepreneur, lui, doit y penser.}

Cette carence, a, en fait, plusieurs explications indépendantes ou combinées :

- définition incomplète du projet avant l'étude de sol, voire modifications importantes postérieures ;

- incompétence du maitre d'œuvre;

- manque de curiosité et/ou de conseil du bureau d'études de sol:

- spécialisation de bureaux d'études de sol plus compétents en géologie qu'en mécanique des sols :

- recherche inconsidérée d'économies par le maître d'ouvrage.

En revanche, on rencontre parfois des bureaux d'études de sols, ayant une attitude très timorée dans les conclusions de leurs investigations.

Parfois, ils se rendent compte que la mission commandée ne permet d'obtenir que partiellement les éléments nécessaires à une parfaite définition dé l'ouvrage.

De plus, pouvant redouter des altérations ponctuelles dans le sol, de mauvaises interprétations, des modifications architecturales avant l'exécution, des travaux de terrassements réalisés sans précaution. ou des travaux confiés à des entreprises peu qualifiées.... ils vont minimiser les hypothèses de portance.

Dans certaines régions de la France, des bureaux d'études de sols font plafonner les contraintes de portance quels que soient les bons résultats donnés par leur essais.

Cette attitude est tout à fait déplaisante, voire choquante pour les entreprises qui se préoccupent de faire le mieux possible leur travail et se voient reléguées au même rang que celles qui ne font pas d'effort.

Néanmoins, dans la majorité des cas, l'entreprise ne doit pas se décourager. Elle doit toujours intervenir pour être plus performante sur le plan économique tout en proposant des bonnes solutions techniques.

Le temps de la ceinture et des bretelles est révolu.

\section{2}

\section{Mise à prix}

Pour illustrer la démarche, nous prendrons le cas de réponse à un appel d'offres pour un marché privé par une entreprise de dimension nationale. Les autre cas bien que spécifiques seront déclinés à partir du schéma de base illustré ci-après.

En principe, le responsable commercial va faire vivre, lui-même ou par un chargé d'affaires, son projet dans l'entreprise.

Avec les éléments qu'elle possède et/ou qu'elle se donne, que ce soit d'une facon interne, semi-interne ou externe, l'entreprise chiffre l'affaire dont les infrastructures en solution de base, et, si elle a des idées en solution variante, afin de faire profiter le maitre d'ouvrage d'une meilleure solution sur le plan économique et par voie de conséquence, de mieux se placer.

Pour atteindre cet objectif, elle fera travailler son bureau d'ètudes qui, à partir des éléments du dossier. fera un avant-projet et dèterminera un dimensionnement et des ratios de structures pour l'ouvrage dont les éléments liés au sol.

C'est souvent de ce bureau d'études que dépend la performance de l'entreprise. C'est lui qui doit avoir les idées et la science pour permettre à l'entreprise de maítriser les projets.

Ensuite, à partir des dimensionnements du béton et des ratios d'armatures, le bureau d'études de prix établit des métrés et constitue grâce aux prix unitaires qu'il possède en bibliothèque ou qu'il a fabrique $\geqslant$, ses déboursés de chantiers.

Ce même bureau d'études de prix sous la maitrisê du chargé d'affaires, va déterminer en collaboration avec le bureau d'études, et les méthodes, la technique d'exécution, le mode opératoire, le planning, et calculer les frais de chantier. Ensuite, la feuille de vente est montée pour déterminer le montant des travaux.

\section{Exécution}

\section{1}

\section{Préparation de chantier}

Une fois le marché signé, en fonction des délais, une période de préparation de chantier démarre ou va démarrer.

Il y a transfert du dossier du commercial vers les travaux.

Le directeur d'exploitation en fonction des contraintes de l'affaire va constituer une équipe d'exécution placée sous l'autorité d'un directeur de travaux.

C'est ce dernier qui prend la responsabilité du chantier et, à ce titre, il prend toutes les décisions nécessaires à la bonne réalisation du projet. 
Il vérifie le dossier qui lui est transféré, il va refaire l'étude de prix qui s'appellera en fonction du vocabulaire propre à l'entreprise, étude d'exécution, étude travaux, point zéro... Un cheminement semblable à celui de l'étude commerciale sera réalisé.

Pour cela, il va faire appel à son bureau d'études, parfois différent du BE qui aura fait l'avant-projet.

En matière d'infrastructures :

- il va vérifier que la solution qu'il a dans son dossier lui convient:

- il contrôle ou fait contrôler que son étude de sol est adaptée et suffisante et, le cas échéant, il sera amené à prendre des dispositions pour la compléter :

- parfois, inspiré par une expêrience précédente, il va étudier le rapport ou le faire étudier par un conseil, afin d'examiner si ses conclusions peuvent être améliorées, en vue d'optimiser les fondations et/ou les soutènements,

En fonction des données économiques du moment, il va peut-être demander des modifications remettant en cause beaucoup de choses apparemment figées. Par exemple il y a deux ans, nous sous-traitions cles pieux à 250 F HT/ml, béton et aciers de liaison compris... cela donnait des idées de variantes !

Par ailleurs, des contraintes de planning, un délai de préparation de chantier réduit, des difficultés pour réaliser l'installation de chantier, des indisponibilités de main-d'cuvre, des conditions particulières d'environnement, un niveau de nappe phréatique élevé... peuvent conduire à sous-traiter les travaux de fondations, particulièrement s'il s'agit de pieux voire de puits.

Dans le cas des dallages et, en particulier, pour les dallages industriels, l'entreprise aura tendance à vouloir sous-traiter ses travaux. En effet, le tour de main du «dallagiste » permet souvent de réaliser les dallages avec plus d'efficience.

Mais un bon entrepreneur sait que sous-traitance r'est pas symonyme d'abandon!

Il faut donc que ces travaux sous-traités soient réalisés en étroite collaboration avec l'entreprise titulaire du lot, qui doit connaitre avec précision ce que va exécuter son sous-traitant et sous quelles conditions.

Par son contrat, l'entreprise se voit parfois imposer des moyens, mais dans tous les cas, elle doit un résultat.

\section{Réalisation}

Avant de couler du béton, il faut procéder aux terrassements généraux, dresser les plates-formes, etc., voire mettre en cuvre un rabattement de nappe...

Dans $90 \%$ des cas, ces travaux préliminaires sont sous-traités.

Là encore, un contrôle rigoureux doit être exercé : - la coupe de sol doit correspondre à celle décrite au rapport ;

- la rencontre de remblais ou de poches limoneuses doit faire l'objet de mesures adaptées prises en concertation avec le bureau de contrôle ;

- le niveau de l'eau sera vérifié ;

- la plate-forme ne fera pas l'objet de remaniements ;
- un dispositif de collecte et d'évacuation des eaux de pluies sera mis en œuvre ;

- etc.

Puis, l'ouvrage est implanté, les axes sont tracés, les chaises sont mises en place.

Dans le cas de fondations par semelles, les « terrassements complémentaires m sont alors exécutés. L'entrepreneur doit vérifier qu'il a atteint la couche de sol sur laquelle il doit se fonder. S'il n'a pas rencontré le bon sol, il doit poursuivre ses terrassements jusqu'à ce qu'il le trouve et. le cas échéant, il se fera assister par son bureau d'études, voire le bureau d'études de sols et le bureau de contrôle pour prendre des dispositions en concertation.

Une fois le niveau atteint, il coulera le béton de propreté ou le gros béton de calage, puis il disposera ensuite ses armatures y compris les attentes pour les verticaux et, enfin, il coulera le béton de semelle.

Dans le cas de fondations profondes, le sous-traitant devra fournir à l'entreprise son « carnet de battage », Celui-ci devra indiquer pour le moins pour chaque pieu, le diamètre d'exécution ( $\geq$ au diamètre théorique), le niveau de plate-forme, l'arase supérieure, sa longueur, l'implantation théorique, le désaxement, l'inclinaison, le type de béton, les armatures.

L'entrepreneur doit vérifier ce qui est visible, notamment le diamètre, l'arase, l'implantation et les armatures en attente. En fonction de ce qu'il relève, il sera parfois amené à faire exécuter de nouveaux pieux supplémentaires, ou à faire des longrines de redressement.

Si des vides de type dissolution de gypse sont à craindre, l'entrepreneur fera exécuter des sondages à l'avancement avec une petite tarière sous chaque pieux, afin d'être assuré de leur assise.

Dans le cas de dallages et, en particulier, pour des dallages de type industriel ou devant recevoir des charges d'exploitations importantes, quel que soit le mode d'exécution, l'entrepreneur sera vigilant sur les points suivants :

- il prendra en compte l'incidence des constructions voisines;

- la plate-forme ne sera pas destructurée:

- les caractéristiques géotechniques de la plate-forme seront vérifiées par l'entreprise;

- la couche de forme sera mise en cuvre avec soin: - le choix du béton sera arrêté de consert entre tous les intervenants:

- la fréquence, l'implantation et la nature des joints seront dessinées sur les plans qui seront suivis;

- les liaisons ou désolidarisations avec des éléments de structures seront clairement représentées sur les plans;

- les armatures seront disposées avec soin et seront calées efficacement.

Ce sont là des points concrets qui peuvent paraître basiques et hors de propos. C'est en réalité le quotidien de l'entrepreneur, qui doit veiller au bon déroulement de toutes les opérations présentant un risque lié au sol.

Celles-ci permettent de respecter le contrat pour la satisfaction du client.

\section{3}

\section{Réception}

En général, le jour de la réception, les ouvrages enterrés ne sont pas l'objet principal de la visite. Toute- 
fois, les sous-sols qui prennent l'eau et les dallages qui fissurent ne passent pas inaperçus !

Pour ces ouvrages liés au sol, directement ou indirectement le sol y est souvent pour quelque chose. L'entrepreneur adopte alors une attitude responsable. Il examine l'incident et recherche une solution avec la maitrise d'ceuvre, le bureau d'études, le bureau d'études de sols et le bureau de contrôle. Il fournit des éléments permettant de rassurer le maitre d'ouvrage pour l'avenir, et procède aux réparations pour lever l'éventuelle réserve.

Dans tous les cas, il fournit au client les plans de récolements.

\section{5}

\section{Formation interne}

Les entrepreneurs qui ont pris conscience de l'importance que la mécanique des sols induit dans leurs ouvrages, se sont d'abord tournés vers des géotechniciens conseils auprès desquels ils leur soumettaient leurs dossiers. Parfois, c'est à l'issue d'un sinistre qu'ils ont rencontré leur " guide s ! D'un conseil qui devenait évaluation et maîtrise de risque, solution constructive, économie.... l'entreprise y a vite trouvé son avantage.

Certains se sont dit qu'il fallait faire partager ce point important avec tous les ingénieurs, conducteurs et directeurs de travaux de l'entreprise, afin de les sensibiliser aux avantages tirés d'une analyse d'un rapport de sol.

Sans vouloir les faire devenir géotechniciens, ils ont mis en place une formation interne dans laquelle interviennent des spécialistes du sol, afin de rendre attentifs tous ces acteurs, pour qu'ils se posent ou n'hésitent pas à poser des questions dans ou hors l'entreprise, afin de tirer la quintessence des hypothèses proposées.

Ainsi, le niveau et la compétence des cadres de l'entreprise ont pu être augmentés, aboutissant à des performances intéressantes et un meilleur service rendu aux clients.

\section{6}

\section{Conclusion}

Bien qu'a priori l'entrepreneur n'ait aucune compétence géologique, il s'est vu attribuer la responsabilité des ouvrages qu'il a réalisés dans et/ou en contact avec le sol.

Du fait que le législateur n’est pas en mesure de reporter la responsabilité du milieu existant devenant support de louvrage à construire sur un autre intervenant que le constructeur dudit ouvrage, il le rend responsable de son support.

Cette situation est en fait bien française, et liée à notre code civil !

Elle n'est donc pas nouvelle. Je pense qu'elle a été et qu'elle est encore vécue comme une contrainte par les entrepreneurs. Pour certains, plutôt que de subir, nous avons pensé qu'il valait mieux essayer de maîtriser le sujet et en tirer parti ! Nous avons donc intégré un peu plus de géotechnique dans notre quotidien, et l'exploitons pour le bien de nos sociétés.

Conscients de l'importance de notre mission, nous avons renforcé notre action pour faire bénéficier nos clients de solutions économiques, et leur assurer néanmoins notre obligation de résultat.

Mais ne nous y trompons pas, je le répète, cette situation est bien française. L'entrepreneur anglosaxon par exemple n'est pas soumis aux mêmes règles! En fait, il n'est qu'exécutant, " couleur de béton, monteur de charpente, remplisseur de vides ») diraient certains ! Ce qu'il réalise, il le fait à partir d'un dossier où tout est défini et figé par l'ingénieriste. Celui-ci conçoit et étudie l'ouvrage et constitue le dossier d'exécution complet. Il fournit dans ce dossier tous les plans dont ceux de fondations à partir d'une étude de sol.

L'entrepreneur qui signe le marché n'a plus qu'à dessiner ses shop drawings pour réaliser ses travaux.

In fine, il n'est responsable que de la borne exécution de ses ouvrages.

Il y a donc encore un bon bout de chemin à parcourir avant d'arriver à une a mondialisation » de la construction ? 